\title{
Performance of the ALICE Inner Tracking System and studies for the upgrade
}

\section{G. Contin*}

Dipartimento di Fisica dell'Univeristà and Sezione INFN, Trieste, Italy

E-mail: giacomo.continets.infn.it

\section{for the ALICE ITS Collaboration}

The Inner Tracking System (ITS) of the ALICE experiment is made out of six layers of silicon detectors exploiting three different technologies (pixel, drift and strip). It covers the central pseudorapidity range of $|\eta|<0.9$ and its distance from the beam line ranges from $\mathrm{r}=3.9 \mathrm{~cm}$ for the innermost pixel layer up to $\mathrm{r}=43 \mathrm{~cm}$ for the outermost strip layer. The main tasks of the ITS are to reconstruct the primary and secondary vertices with high resolution; to track and identify charged particles with a low $\mathrm{p}_{t}$ cut-off as a standalone tracker; to improve the momentum and angle resolution for tracks reconstructed in the outside tracking detectors. The performance of the ITS in p-p and $\mathrm{Pb}-\mathrm{Pb}$ collisions in 2010 are presented both from the hardware point of view, with a brief overview of the features of the system, and the physics achievements for what concerns the vertexing, the tracking and the particle identification. Furthermore, an outlook is given on a possible upgrade of the ALICE ITS which is presently being studied, in order to extend its physics performance by improving the measurements of charmed hadrons and accessing new physics items like the measurement of the beauty hadrons.

10th International Conference on Large Scale Applications and Radiation Hardness of Semiconductor Detectors,

July 6-8, 2011

Firenze Italy

\footnotetext{
*Speaker.
} 


\section{Introduction}

The ALICE experiment at LHC is dedicated to the observation of heavy ion collisions and aims at studying the behavior of the strongly-interacting matter in conditions of high energy density (> $\left.10 \mathrm{GeV} / \mathrm{fm}^{3}\right)$ and high temperature $(\gtrsim 200 \mathrm{MeV}$ ). In addition, ALICE developed a detailed p-p collision program which serves as a reference for the heavy ion program and on the other hand complements the programme of the dedicated $p$-p experiments, thanks to the low transverse momentum cut-off, lower than $100 \mathrm{MeV} / c$, the excellent PID capability and the efficient minimum bias trigger.

The ALICE detector is composed of two main sections: a central barrel, covering the full azimuth in the acceptance region $|\eta|<0.9$, and a forward $(2.5<\eta<4)$ muon arm [1]. The high-multiplicity heavy ion environment requires the central barrel to perform a robust tracking: every track is reconstructed using up to 165 three-dimensional points by the Inner Tracking System (ITS) and the Time Projection Chambers (TPC) over a wide transverse momentum range (from $100 \mathrm{MeV} / c$ up to $100 \mathrm{GeV} / c)$. The large lever arm provided by the outer radius of the TPC ( $2.5 \mathrm{~m}$ ) guarantees a good tracking resolution at high $p_{t}$, while the total material budget has been minimized $\left(\sim 13 \% X_{0}\right)$ in order to reduce the multiple scattering effects. The identification of the charged particles (PID) by the ALICE detector is carried out over the mentioned $p_{t}$ range and is performed through different techniques: $\mathrm{d} E / \mathrm{d} x$, time-of-flight, transition radiation and Cherenkov radiation.

In this proceeding we present the ITS performance in $2010 \mathrm{p}-\mathrm{p}$ and $\mathrm{Pb}-\mathrm{Pb}$ collisions. We start by describing the ITS tasks and characteristics in Section 2 and the hardware features of its three sub-systems in Section 3 . The ITS performance in terms of tracking, vertex reconstruction, impact parameter resolution and PID are addressed in Section 4 . An outlook on the studies for a possible upgrade for the ITS is given in Section 5 .

\section{System overview}

The main tasks of the ITS are to localize the primary vertices with a resolution better than $100 \mu \mathrm{m}$, to reconstruct the secondary vertices from hyperons and $\mathrm{B}$ and $\mathrm{D}$ meson decays, to track and identify charged particles with $p_{t}<200 \mathrm{MeV} / c$ or particles traversing the dead zones of the TPC and to improve the momentum and angle resolution for particles tracked by the TPC. Placed close to the interaction point, the ITS (see fig. 1, left) is composed of 6 cylindrical layers of silicon detectors using three different technologies: pixel, drift and strip. The system is conceived to handle a high particle density, up to 8000 per unit of pseudo-rapidity, with very good spatial precision in the bending direction, down to $12 \mu \mathrm{m}$ for the innermost layers, high efficiency and granularity. The design minimizes the distance of the first layer from the interaction point, introduces a limited material budget and allows measuring the $\mathrm{d} E / \mathrm{d} x$ for the particle identification in the low $p_{t}$ region.

The ITS layer radii range from $3.9 \mathrm{~cm}$ (innermost pixel layer) up to $43 \mathrm{~cm}$ (outermost strip layer), covering the $|\eta|<0.9$ pseudo-rapidity range. The $\sim 9.8$ million channels of the two innermost layers can handle the high density of particles with a maximum occupancy of $2.1 \%$. The total material budget introduced by the whole ITS is $\sim 8 \% X_{0}$. The geometry and the material 

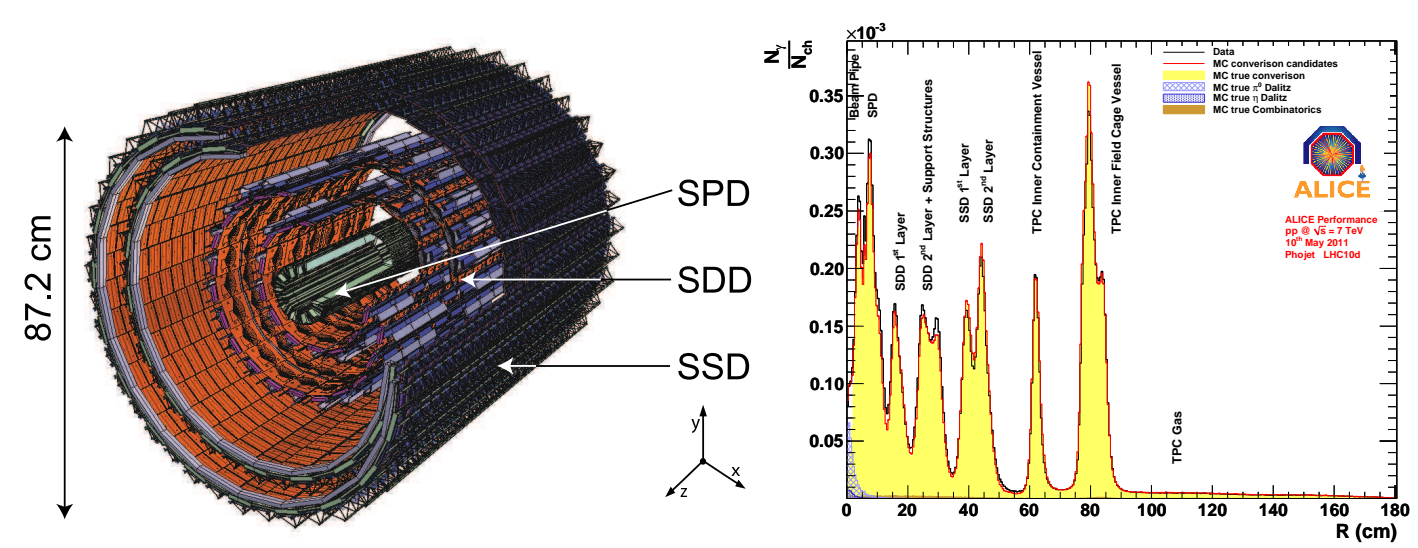

Figure 1: Layout of the ITS and definition of the ALICE global reference system (left). Contribution of the ITS layers to the gamma conversion distribution from data and Monte Carlo simulation in $\sqrt{s}=7 \mathrm{TeV}$ p-p collisions (right).

budget are accurately described in the Monte Carlo simulation, as shown by the comparison with the actual reconstructed photon conversion in the material in fig. 11(right).

\section{ITS hardware features}

The two innermost layers are composed of Silicon Pixel Detectors (SPD). The basic SPD building block is a module consisting of a two-dimensional sensor matrix of reverse-biased silicon detector diodes, $200 \mathrm{~mm}$ thick, bump-bonded to 5 front-end chips, thinned down to $150 \mathrm{~mm}$. The sensor matrix consists of $256 \times 160$ cells, each measuring $50 \mu \mathrm{m}(r \phi)$ by $425 \mu \mathrm{m}(z)$. The two layers have a length of $28 \mathrm{~cm}$ and radii of around 4 and $8 \mathrm{~cm}$, respectively, mounted around the beam pipe; the system is cooled down with a $\mathrm{C}_{4} \mathrm{~F}_{10}$-based evaporative cooling that allows operating at room temperature. The SPD performs a fast two-dimensional read-out in $256 \mu \mathrm{s}$. The output signal is digital, and a logical 1 is given when the signal surpasses the threshold, tuned at the pixel level. It can contribute to the L0-trigger through the Fast-OR logic [2]: the off-detector SPD trigger system elaborates in real time the 1200 Fast-OR signals, each of them is asserted by a readout chip on the presence of at least one pixel hit in the matrix. The Fast-OR trigger contribution is especially used in $\mathrm{p}-\mathrm{p}$ collision to improve sensibly the background rejection and in $\mathrm{Pb}-\mathrm{Pb}$ collisions for the event selection.

The two intermediate layers contain Silicon Drift Detectors (SDD). One SDD module consists of a drift detector and its front-end electronics. The sensitive area of a detector is split into two drift regions, where electrons move in opposite directions, by a central cathode. A second bias supply keeps the biasing of the collecting region independent of the drift voltage. Each drift region is equipped with 256 anodes to collect the charges, and three rows of 33 MOS charge injectors used to monitor the drift velocity, which is very sensitive to the temperature. The two-dimensional points are reconstructed exploiting the anode segmentation in one direction and the drift time of the charges in the other one. The SDD front-end electronics is based on three types of ASICs, two bonded on the sensor and one located at each end of the ladder. The sampling rate can be set via 
software at 20 or $40 \mathrm{MHz}$. A water cooled system is used to take away the power dissipated by the front-end electronics and to maintain a temperature stability of $<0.1 \mathrm{~K}$. It is a combination of two independent underpressure water circuits, one coupled to the front-end electronics via the pipes running along the ladders and the other coupled to the readout electronics, via pipes embedded in the end-ladder structure [3].

Silicon Strip Detectors (SSD) are used on the two outermost layers of the ITS. The SSD is composed of 1698 modules mounted on carbon-fiber supports, each one consisting of a 1536-strip double-sided silicon sensor connected through aluminum-kapton microcables to twelve HAL25 front-end chips, for a total number of $\sim 2.6$ million read-out channels [ $₫$. The strip pitch (95 $\mu \mathrm{m})$ and the relative $\mathrm{P}$ - N-side stereo angle inclination (35 mrad) allow the SSD to provide twodimensional measurement of the track position, reaching an intrinsic point resolution of $20 \mu \mathrm{m}$ and $800 \mu \mathrm{m}$ in the $r \phi$ and $z$ directions, respectively, and a good ghost point rejection: the fraction of non-ambiguous impact points is above 98\%, with the multiplicities observed in $\mathrm{Pb}-\mathrm{Pb}$ collisions. Each front-end chip contains 128 identical channels. It preamplifies, shapes and stores analogue signals from the sensor and provides an interface to the readout system. The analogue readout has a large dynamic range that allows using the $\mathrm{d} E / \mathrm{d} x$ measurement for the particle identification in the low momentum region (up to $1 \mathrm{GeV} / c$ for protons). The thickness of the SSD, including support and services, corresponds to $2.2 \% X_{0}$.

\section{Physics performance in $\mathrm{p}-\mathrm{p}$ and $\mathrm{Pb}-\mathrm{Pb}$}

The two-dimensional points reconstructed in the six ITS layers are used to track the charged particles in the central barrel $(|\eta|<0.9)$, both in combination with the other ALICE sub-detectors (global tracking) and in the ITS detector alone (ITS-standalone tracking). The global track reconstruction is performed in the following steps. The first one is the computation of the primary vertex using SPD tracklets, i.e. pairs of aligned SPD hits (one per layer). Track reconstruction then starts in the TPC (inward) where the track density is minimal, using the outermost pad rows and the computed primary vertex position as seed, and continues in the ITS (inward), matching TPC reconstructed tracks to the SSD layers and following them down to the innermost SPD layer. The next step is track back-propagation, to the outermost layer of the ITS, to the outermost radius of the TPC and to the outer detectors (TRD, TOF, HMPID, PHOS, EMCal) for PID. A refit is then performed inward in TRD, TPC, ITS, the tracks are propagated to the primary vertex reconstructed in the first step and the track parameters are refined. Finally the primary vertex is recalculated with optimal resolution using reconstructed tracks.

The ITS-standalone tracking is aimed at extending the $p_{t}$ acceptance down to $\sim 100 \mathrm{MeV} / c$, reconstructing the high-momentum tracks going entirely inside the dead zones between the TPC sectors and decaying between the ITS and TPC. The ITS-standalone tracking is normally performed on the remaining points after the standard TPC+ITS track reconstruction [5]. The prolongation efficiency between the TPC and ITS, measured in Pb-Pb collisions at $\sqrt{s_{N N}}=2.76 \mathrm{TeV}$ during the 2010 data acquisition, is reported as a function of transverse momentum in fig. Q, left. It is flat, above $95 \%$, for transverse momenta below $1 \mathrm{GeV} / c$, decreasing to $90 \%$ at higher momenta, if at least two points in the ITS are matched by the TPC track. A 10\% drop is measured uniformly over the considered range if at least one point on the SPD layers is further requested. The right panel 

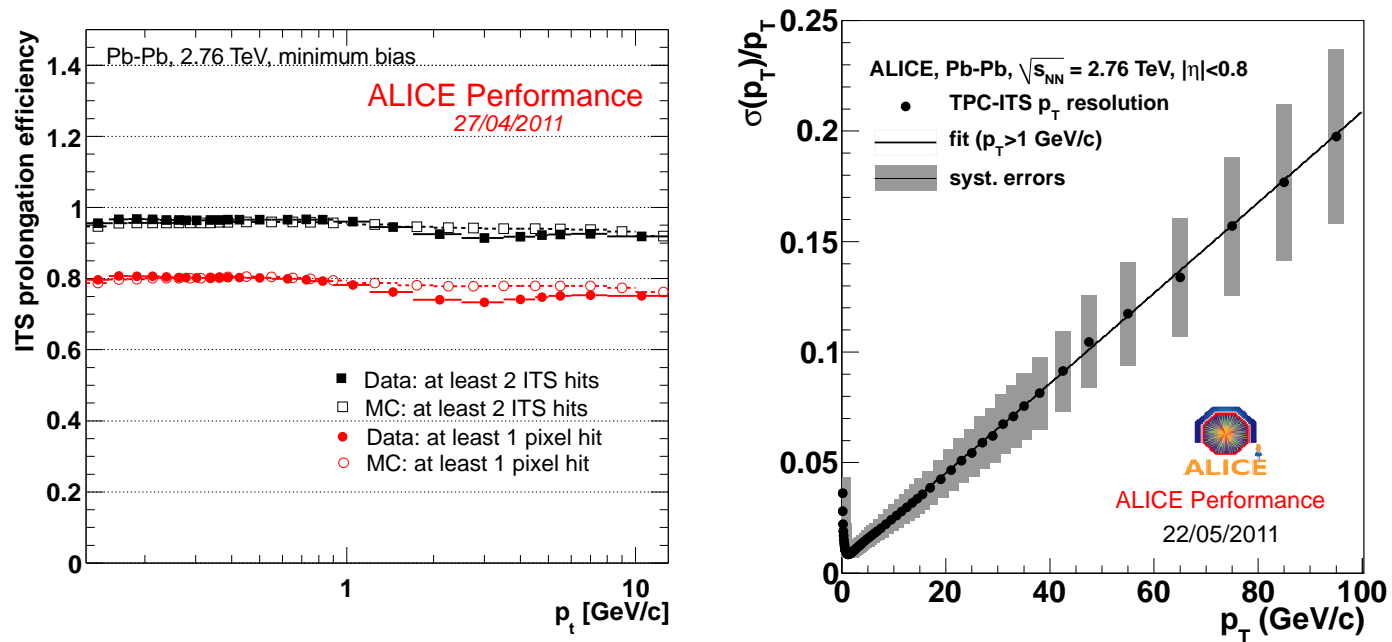

Figure 2: Left panel: track prolongation efficiency from TPC to ITS as a function of the transverse momentum in $\sqrt{s}=7 \mathrm{TeV}$ p-p data, for the request of at least two points in the ITS (black) and the further request of at least one in SPD (red); empty points represent simulation. Right panel: transverse momentum resolution of the TPC+ITS combined tracking.

shows the transverse momentum resolution as a function of the transverse momentum: better than $5 \%$ below $1 \mathrm{GeV} / c$, it grows linearly with $p_{t}$ up to $\sim 20 \%$ at $100 \mathrm{GeV} / c$; the $p_{t}$ dependence of the systematic error reflects the unfolding uncertainty at high $p_{t}$. The ITS-standalone tracking shows a $p_{t}$ resolution better than $6 \%$ for pions in the range $200-800 \mathrm{MeV} / c$ and can extend the acceptance of the pions down to $80-100 \mathrm{MeV} / c$.

The primary vertex of the interaction in ALICE is reconstructed through two different methods, as mentioned before: at first, all the tracklets selected within a fiducial window in $(\Delta \eta, \Delta \phi)$ are combined to extract the vertex position (tracklets method). This method is used to monitor the interaction position quasi-online, to initiate the tracking and to measure important first-physics observables, as for example the charged particle multiplicity density at midrapidity [6]. At the end of the tracking procedure, a second method based on the straight line approximation of the reconstructed tracks in the vicinity of the vertex is used (tracks method). This method reaches a better resolution over the whole tracklets multiplicity range, as shown in fig. 目(left). For high multiplicity events, where the effect of the intrinsic vertex resolution method is assumed to be negligible for the tracks, the residual resolution limit estimates the luminous region size, i.e. the spatial region where collisions take place. In $\mathrm{Pb}-\mathrm{Pb}$ collisions, the vertex resolution is estimated by dividing the track sample into two random sub-samples and reconstructing two vertices for the two samples. The difference between these two vertices is the measurement of the resolution as a function of the half event tracklet multiplicity (fig $\mathcal{B}$, right panel). The extrapolated resolution is less than $10 \mu \mathrm{m}$ for the 5\% most central events.

A benchmark parameter to assess the ITS performance is the resolution of the track impact parameter in the transverse plane, which is the convolution of the vertex resolution and the track pointing resolution. Defined as the distance between the projection of a track in the bending plane 

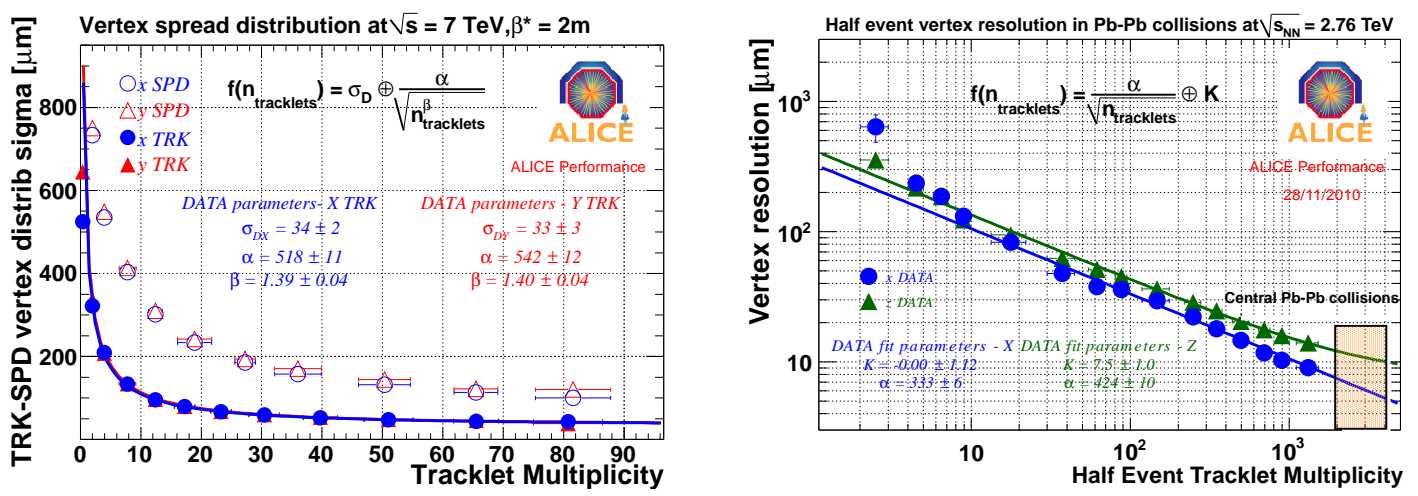

Figure 3: Left panel: primary vertex reconstructed with tracks and with SPD tracklets only: spread of vertices in $\sqrt{s}=7 \mathrm{TeV}$ p-p data. For high multiplicity events, the resolution seen for the vertices reconstructed with tracks (filled markers) estimates the luminous region size. Right panel: vertex resolution in $\mathrm{Pb}-\mathrm{Pb}$ collisions at $\sqrt{s_{N N}}=2.76 \mathrm{TeV}$ as a function of half of the tracklets multiplicity of the event. The resolution is estimated through the two-samples method (see text for details) and is extrapolated for the events in the $0-5 \%$ centrality class (orange box).

and the reconstructed position of the primary vertex, $d_{0}(r \phi)$ is the reference variable to look for tracks from the decay vertices: the impact parameter resolution is shown in fig. \# (left), in agreement with the Monte Carlo simulation within $10 \%$. The multiple scattering in the material degrades the ITS performance at low $p_{t}$, while the impact parameter resolution is better than $65 \mu \mathrm{m}$ for particles with $p_{t}>1 \mathrm{GeV} / c$; in the asymptotic limit the impact parameter resolution is driven by the $p_{t}$ independent detector resolution of the layers, which accounts for the hit position uncertainty on the sensor and the detector misalignment. The ITS-standalone mode enables the tracking for very low momentum particles down to $80 \mathrm{MeV} / c$ for pions.

As mentioned before, the ITS identifies low-momentum particles in the non-relativistic region measuring the energy loss in the SDD and SSD silicon. The four analogue measurements of the charge collected in these four layers are corrected for the path length after the tracking and the truncated mean method is applied to account for the long tail towards high energy loss values. The particle is then identified from the measured $p_{t}$ and energy loss: two particle species are considered to be separated if the means of the corresponding gaussian $\mathrm{d} E / \mathrm{d} x$ distributions, filled after applying the truncated mean method, differ by at least 3 standard deviations [7]. In fig. 母(right) the bands of kaons, pions and protons detected in $\mathrm{Pb}-\mathrm{Pb}$ collisions are clearly visible and centered around the continuous lines representing the theoretical curves from the Bethe-Block formula for the average energy loss. The achieved resolution in the energy measurement is $\sim 10 \div 15 \%$ and the ITS allows separating kaons from protons up to $1 \mathrm{GeV} / \mathrm{c}$ and pions from kaons up to $450 \mathrm{MeV} / c$, covering also the region below $100 \mathrm{MeV} / c$, outside of TPC and TOF particle identification capability. The cross-contamination between different particle species is below $10 \%$.

\section{Outlook on the ITS upgrade plans}

After two years of operation with p-p collisions and the first run with $\mathrm{Pb}-\mathrm{Pb}$ collisions, the 

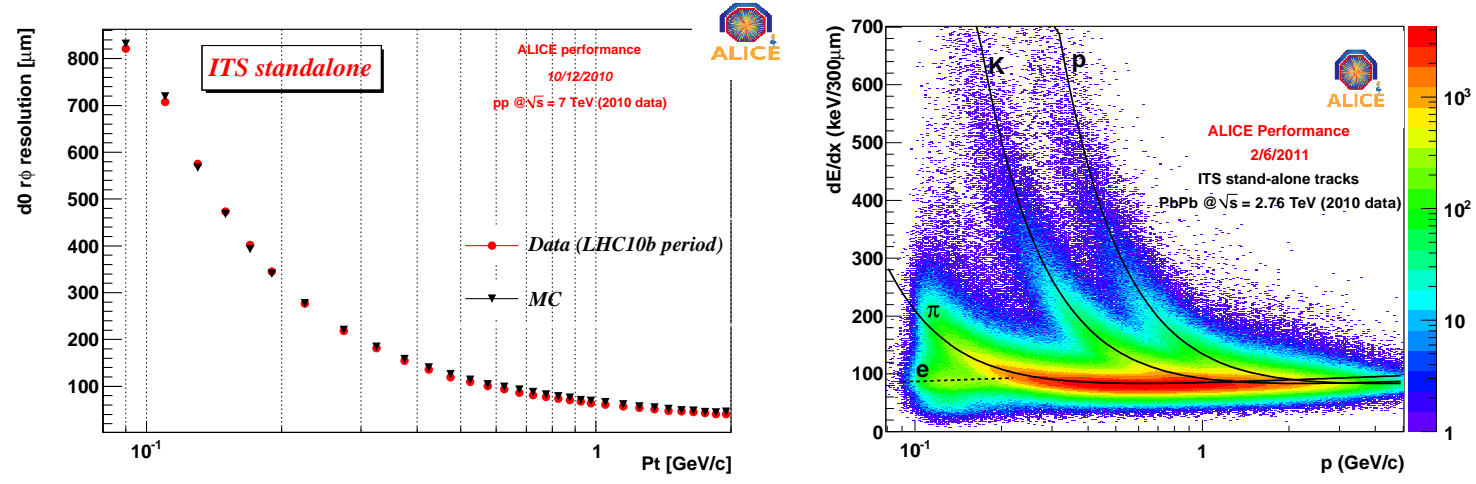

Figure 4: Transverse impact parameter resolution as a function of $p_{t}$ for the tracks reconstructed in the p-p collisions at $7 \mathrm{TeV}$ by the ITS standalone (left) and compared with the Monte Carlo results. The $\mathrm{d} E / \mathrm{d} x$ of charged particles as a function their momentum, both measured by the ITS standalone, in $\mathrm{Pb}-\mathrm{Pb}$ collisions at $2.76 \mathrm{TeV}$ (right).

ALICE ITS has already demonstrated its excellent capabilities. Nevertheless there are still several topics for which the current detector is not fully optimized and where the physics performance could be significantly improved. In particular, the possibility to extend the charmed baryon decay study and to access the exclusive measurement of beauty hadrons through an upgrade of the ITS is being studied by the ALICE ITS Collaboration [8]. One of the possibilities for a fully upgraded ITS will consist of 7 layers of silicon tracking detectors covering the region from the beam pipe up to $450 \mathrm{~mm}$. It is expected that the radius of the innermost layer will be reduced to $20 \mathrm{~mm}$ requiring increased radiation tolerance. Furthermore, there is a strong effort to minimize the material budget of the inner layers in order to improve the impact parameter resolution: this can be achieved by using monolithics pixel detectors or hybrid pixel detectors with thinned chip and sensor, and minimizing the contribution coming from supports and services. The three inner layers, where the track density is higher, must also provide unambiguous $2 \mathrm{D}$ information to allow efficient event reconstruction. Therefore, they will be equipped with high granularity pixel detector. Both hybrid and monolithic pixels are under consideration. For the hybrid option, the target thickness is 100 $\mu \mathrm{m}$ for the sensor chip plus $50 \mu \mathrm{m}$ for the ASIC, with a pixel area of the order of $30 \times 100 \mu \mathrm{m}^{2}$; monolithic pixels typically feature a thickness of $50 \mu \mathrm{m}$ and pixel dimensions of $20 \times 20 \mu \mathrm{m}^{2}$. The outer layers could be equipped either with monolithic pixels or with more conventional silicon strip sensors. Silicon strips are a proven and mature technology and they will allow maintaining the $\mathrm{dE} / \mathrm{dx}$ capability that the current detector provides. In addition, a new design with shorter strips (20 mm with respect to the actual $40 \mathrm{~mm}$ ) arranged in two rows on each sensor side is being considered: the increased granularity achieved with the new layout will allow operating the detector even at shorter distance from the interaction point, therefore in higher particle density conditions.

Different ITS upgrade scenarios have been proposed and implemented in the simulation to estimate the impact of refined detector parameters on the physics performance. The heavy-flavour detection relies in particular on the precision in the determination of the track impact parameter and the capability of resolving the slightly displaced secondary vertex of the decays: the simulation 

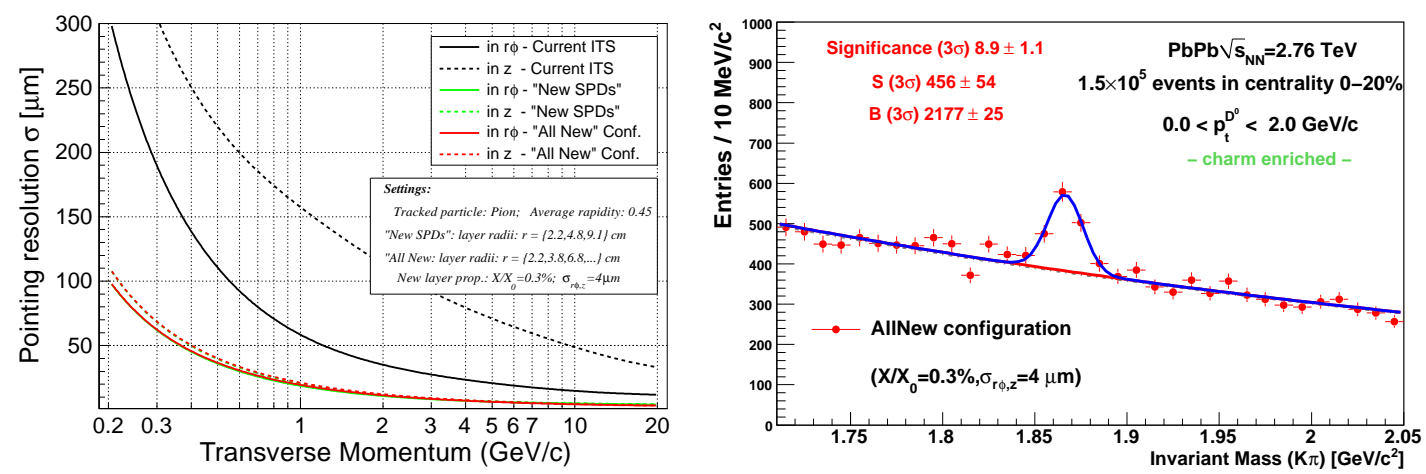

Figure 5: Left: pion pointing resolution as a function of $p_{t}$ for the current ITS (black curves) and for two upgrade scenarios (red and green). Right: invariant mass distributions of $\mathrm{D}_{0}$ candidates in simulated $\sqrt{s_{N N}}=$ 2.76 TeV Pb-Pb events, obtained for $0<p_{t}<2 \mathrm{GeV} / c$ in the event centrality range $0-20 \%$ with the upgraded ITS.

reported in fig. 5 (left) shows the track pointing resolution improvement (a factor 2-3) that can be achieved both in the $z$ and in the $r \phi$ direction by replacing the present ITS (black curves) with the seven new silicon layers of the upgraded ITS scenario (red curves). For example, as a benchmark for all the charmed meson analyses, the signal extraction of the $\mathrm{D}_{0}$ meson significantly benefits of the improved track impact parameter resolution and thus of the better separation between signal and background. Fig. 5, right panel, shows the invariant mass distribution obtained from the Monte Carlo sample in the upgraded ITS scenario for $0<p_{t}<2 \mathrm{GeV} / c$ in the event centrality range 0 $20 \%$ : the improved background rejection allows the extraction of a clear $\mathrm{D}_{0}$ signal, that cannot be seen with the current ITS.

\section{Summary}

In the $2010 \mathrm{p}-\mathrm{p}$ and $\mathrm{Pb}-\mathrm{Pb}$ runs the Inner Tracking System of the ALICE experiment demonstrated its capabilities, meeting the design requirements. It contributed significantly to the event reconstruction, matching more than $90 \%$ of the tracks reconstructed by the TPC and improving the momentum and angle resolutions. In standalone mode it reconstructed the tracks missed by the TPC with good momentum resolution, extending the $\mathrm{p}_{t}$ range below $100 \mathrm{MeV} / c$. The ITS provided also a prompt reconstruction of the interaction primary vertex, achieving a resolution of $10 \mu \mathrm{m}$ for the 5\% most central $\mathrm{Pb}-\mathrm{Pb}$ events, and well separated it from secondary vertices from hyperons and heavy-flavour meson decays, thanks to the achieved impact parameter resolution. The energy loss measurement allowed to identify charged particles down to less than $100 \mathrm{MeV} / c$. The ITS is therefore playing a crucial role in the ALICE analyses. In order to improve the ITS physics performance and in particular to extend heavy quark studies to the decays of charmed baryons and beauty hadrons, a possible upgrade of the ITS has been proposed. The achievable performance are being studied through Monte Carlo simulations and the technology options are being extensively investigated and tested. 


\section{References}

[1] The ALICE Collaboration, The ALICE experiment at the CERN LHC, 2008 JINST 3 S08002

[2] V. Altini for the ALICE Collaboration, Trigger performance of the ALICE silicon pixel detector, 2011 Nucl. Instrum. Meth. A $\mathbf{6 5 0} 30$.

[3] S. Coli et al., The cooling system of the Silicon Drift layers of the ALICE Inner Tracking System, ALICE Internal Note ALICE-INT-2008-008

[4] M. Bregant et al., The ALICE vertex detector: Focus on the micro-strip layers, 2006 Nuclear Instruments and Methods in Physics Research Section A Volume 569, Issue 1, p. 29-32

[5] The ALICE Collaboration, ALICE: Physics Performance Report, Volume II, 2006 J. Phys. G: Nucl. Part. Phys. 321295

[6] K. Aamodt et al. (ALICE Collaboration), Charged-particle multiplicity density at mid-rapidity in central Pb-Pb collisions at $\sqrt{s_{N N}}=2.76$ TeV, 2010 Phys. Rev. Lett. 105, 252301

[7] Alexander Kalweit for the ALICE Collaboration, Particle identification in the ALICE experiment, 2011 J. Phys. G: Nucl. Part. Phys. 38, 124073

[8] The ALICE Collaboration, Conceptual Design Report for the Upgrade of the ALICE ITS, CERN-LHCC-2012-005, LHCC-G-159 